\title{
Solution of the Fractional Black-Scholes Option Pricing Model by Finite Difference Method
}

\author{
Lina Song and Weiguo Wang \\ Center for Econometric Analysis and Forecasting, School of Mathematics and Quantitative Economics, \\ Dongbei University of Finance and Economics, Dalian 116025, China
}

Correspondence should be addressed to Lina Song; sln_dufe@hotmail.com

Received 17 March 2013; Revised 2 June 2013; Accepted 16 June 2013

Academic Editor: Changbum Chun

Copyright (c) 2013 L. Song and W. Wang. This is an open access article distributed under the Creative Commons Attribution License, which permits unrestricted use, distribution, and reproduction in any medium, provided the original work is properly cited.

\begin{abstract}
This work deals with the put option pricing problems based on the time-fractional Black-Scholes equation, where the fractional derivative is a so-called modified Riemann-Liouville fractional derivative. With the aid of symbolic calculation software, European and American put option pricing models that combine the time-fractional Black-Scholes equation with the conditions satisfied by the standard put options are numerically solved using the implicit scheme of the finite difference method.
\end{abstract}

\section{Introduction}

The theory and methodology of partial differential equation started to become popular to study option pricing problems, after the classical Black-Scholes equation was proposed. The available research results include mainly two aspects: one is to give values of options using more powerful numerical and analytic methods; the other is to derive new pricing models that reflect the actual financial market more closely.

The Black-Scholes equation has been increasingly attracting interest over the last two decades since it provides effectively the values of options. But the classical Black-Scholes equation was established under some strict assumptions. Therefore, some improved models have been proposed to weaken these assumptions, such as stochastic interest model [1], Jump-diffusion model [2], stochastic volatility model [3], and models with transactions costs $[4,5]$. With the discovery of the fractal structure for financial market, the fractional Black-Scholes models [6-9] are derived by replacing the standard Brownian motion involved in the classical model with fractional Brownian motion. These fractional BlackScholes models are still partial differential equations with integer order derivative, which are reduced to the classical Black-Scholes equation if we let the Hurst exponent $H=$ $1 / 2$. The differential equation involving derivatives of fractional order is a powerful tool for studying fractal geometry and fractal dynamics. As a generalization of the integerorder differential equation, fractional differential equation is used to model important phenomena in various fields such as fluid flow, electromagnetic, acoustics, electrochemistry, cosmology, and material science. Recently, fractional partial differential equation was introduced more and more into financial theory. Wyss [10] gave the fractional Black-Scholes equation with a time-fractional derivative to price European call option. Cartea and del-Castillo-Negrete [11] gave several fractional diffusion models of option prices in markets with jumps and priced barrier option using fractional partial differential equation. Jumarie $[12,13]$ derived the time- and space-fractional Black-Scholes equations and gave optimal fractional Merton's portfolio. The aim of this paper is to try to combine Jumarie's time-fractional Black-Scholes equation with the terminal and boundary conditions satisfied by the standard put options to study the pricing problems for European and American put options.

In the present work, the option price $V=V(S, t)$ is suggested to be subject to the time-fractional Black-Scholes equation $[12,13]$ with the following form:

$$
\begin{array}{r}
\frac{\partial^{\alpha} V}{\partial t^{\alpha}}=\left(r V-r S \frac{\partial V}{\partial S}\right) \frac{t^{1-\alpha}}{\Gamma(2-\alpha)}-\frac{\Gamma(1+\alpha)}{2} \sigma^{2} S^{2} \frac{\partial^{2} V}{\partial S^{2}}, \\
t>0,0<\alpha \leq 1,
\end{array}
$$


where $r, \sigma$ denote risk-free interest rate and volatility, respectively. $\partial^{\alpha} V / \partial t^{\alpha}$ involved in (1) is a modified Riemann-Liouville fractional derivative [12-15], which is defined using the equality

$$
\begin{aligned}
D_{t}^{\alpha} f(t) & =\lim _{h \rightarrow 0} \frac{\Delta^{\alpha} f(t)}{h^{\alpha}} \\
& =\lim _{h \rightarrow 0} \sum_{k=0}^{\infty}(-1)^{k}\left(\begin{array}{l}
\alpha \\
k
\end{array}\right) f(t+(\alpha-k) h), \quad 0<\alpha \leq 1
\end{aligned}
$$

and can be represented as

$$
\begin{aligned}
& D_{t}^{\alpha} f(t) \\
& =\left\{\begin{array}{r}
\frac{1}{\Gamma(-\alpha)} \int_{0}^{t}(t-\xi)^{-\alpha-1}(f(\xi)-f(0)) d \xi, \\
\text { if } \alpha<0, \\
\frac{1}{\Gamma(1-\alpha)} \frac{d}{d t} \int_{0}^{t}(t-\xi)^{-\alpha}(f(\xi)-f(0)) d \xi, \\
\text { if } 0<\alpha<1,
\end{array}\right. \\
& \left(f^{(\alpha-n)}(t)\right)^{(n)}, \quad \text { if } n \leq \alpha<n+1, n \geq 1 .
\end{aligned}
$$

The value of European put option is taken as a solution of (1) with the following terminal and boundary conditions:

$$
\begin{gathered}
V(S, T)=\max (K-S, 0), \\
V(0, t)=K \exp (-r(T-t)), \\
V(S, t) \longrightarrow 0, \quad S \longrightarrow+\infty .
\end{gathered}
$$

Pricing European put option based on (1) and (4) can be implemented by finite differences approximation. For American put option, this idea is operable. The main problem for pricing an American put option is to consider the possibility of early exercise. To avoid arbitrage, the option value at each point in the $(S, t)$ space cannot be less than the intrinsic value [16]. For that, the principle of dynamic programming (at a given time, the optimal strategy corresponds to the maximum of either the exercise value or the value associated with selecting an optimal strategy an instant later) [17] expressed by the following equation is applied, which can be implemented using finite difference method and successive overrelaxation (SOR) method

$$
V(S, t)=\max (K-S, V(S+d S, t+d t)) .
$$

Fractional Black-Scholes equation (1) is actually a special kind of fractional advection-dispersion equation, which has time-varying coefficient and time-fractional derivative. A general fractional advection-dispersion equation has been studied in many works in the literature. Sousa [18] employed a second-order explicit finite difference method derived by a Lax-Wendroff-type time discretization procedure to solve space-fractional advection-diffusion equation. Mohebbi and Abbaszadeh [19] applied compact finite difference scheme to deal with time-fractional advection-dispersion equation with constant coefficient. Meerschaert and Tadjeran [20] developed explicit and implicit Euler methods for the spacefractional advection-dispersion equation. Liu et al. [21] developed effective numerical methods for solving several spacetime fractional advection-dispersion equations. Liu et al. [22] used explicit and implicit difference methods to solve spacetime fractional advection-dispersion equation and proved the stability and convergence of the methods. More powerful methods, such as implicit MLS meshless method [23], Adomian decomposition method [24], homotopy perturbation method [25], and spectral regularization method [26], have been used to solve numerical and analytical solutions for various types of fractional advection-dispersion equation. In this paper, we combine time-fractional Black-Scholes equation (1) with conditions satisfied by European and American put options to construct put option pricing models. It is obvious that this fractional derivative model can be reduced to the classical Black-Scholes model if the derivative order $\alpha=1$. As a generalization, this time-fractional pricing model of European and American put options is solved numerically using the implicit finite difference technique.

\section{Numerical Scheme}

Numerical and analytical methods [16-43] are used to study a great quantity of differential equations. Among them, the finite difference method is a direct and effective numerical algorithm. With this technique, the differential equation is transformed into a difference equation by the discretization of derivatives, and numerical solutions are finally obtained. The approach has been extended successfully to deal with various fractional differential equation [18-22, 38-43]. In this section, the implicit finite difference mode is given.

Take the change of variable

$$
\tau=T-t
$$

Equation (1) can be transformed into the following form:

$$
\begin{gathered}
\tau^{(\alpha-1)}(T-\tau)^{(1-\alpha)} \frac{\partial^{\alpha} V}{\partial \tau^{\alpha}}+\left(r V-r S \frac{\partial V}{\partial S}\right) \frac{(T-\tau)^{1-\alpha}}{\Gamma(2-\alpha)} \\
-\frac{\Gamma(1+\alpha)}{2} \sigma^{2} S^{2} \frac{\partial^{2} V}{\partial S^{2}}=0
\end{gathered}
$$

In order to use finite difference approximation, we start by $S \in\left[0, S_{\max }\right]$ and $\tau \in[0, T)$. Let $h=S_{\max } / M, k=T / N$ $\left(M, N \in Z_{+}\right)$be the grid sizes in space and time; the computational domain is discretized by a uniform grid $\left(S_{m}, \tau_{n}\right)$ with $S_{m}=m h(m=0,1, \ldots, M)$ and $\tau_{n}=n k(n=0,1, \ldots, N)$, where $S_{M}=S_{\max }$ is a realistic and practical approximation to infinity. $V_{m}^{n}$ denotes an approximate solution of (7) in $S_{m}$ at the time $\tau_{n}$.

As pointed out in $[12,13]$, Jumarie's definition and the so-called Caputo's definition yield the same result when the function is differentiable. So, we take Caputo finite difference approximation [38] for the modified RiemannLiouville fractional derivative involved in (7), namely,

$$
\frac{\partial^{\alpha} V}{\partial \tau^{\alpha}}=\varrho_{\alpha, k} \sum_{j=1}^{n} \omega_{j}^{(\alpha)}\left(V_{m}^{n-j+1}-V_{m}^{n-j}\right)+O(k),
$$


where $\varrho_{\alpha, k}=1 / \Gamma(2-\alpha) k^{\alpha}$ and $\omega_{j}^{(\alpha)}=j^{1-\alpha}-(j-1)^{1-\alpha}$ that satisfies (i) $\omega_{j}^{(\alpha)}>0$, (ii) $\omega_{j}^{(\alpha)}>\omega_{j+1}^{(\alpha)}[22,38]$.

For spatial derivative, we use the following difference approximation:

$$
\begin{gathered}
\frac{\partial V}{\partial S}=\frac{V_{m+1}^{n}-V_{m-1}^{n}}{2 h}+O(h), \\
\frac{\partial^{2} V}{\partial S^{2}}=\frac{V_{m+1}^{n}-2 V_{m}^{n}+V_{m-1}^{n}}{h^{2}}+O\left(h^{2}\right) .
\end{gathered}
$$

Substituting (8) and (9) to (7) can derive

$$
\begin{gathered}
\tau^{(\alpha-1)}(T-\tau)^{(1-\alpha)} \varrho_{\alpha, k} \sum_{j=1}^{n} \omega_{j}^{(\alpha)}\left(V_{m}^{n-j+1}-V_{m}^{n-j}\right) \\
+\left(r V_{m}^{n}-r m h \frac{V_{m+1}^{n}-V_{m-1}^{n}}{2 h}\right) \\
\times \frac{(T-n k)^{1-\alpha}}{\Gamma(2-\alpha)}-\frac{\Gamma(1+\alpha)}{2} \\
\times \sigma^{2}(m h)^{2} \frac{V_{m+1}^{n}-2 V_{m}^{n}+V_{m-1}^{n}}{h^{2}}=0
\end{gathered}
$$

or

$$
\begin{gathered}
\tau^{(\alpha-1)}(T-\tau)^{(1-\alpha)} \varrho_{\alpha, k} \sum_{j=1}^{n} \omega_{j}^{(\alpha)}\left(V_{m}^{n-j+1}-V_{m}^{n-j}\right) \\
+\left(r V_{m}^{n}-r m \frac{V_{m+1}^{n}-V_{m-1}^{n}}{2}\right) \\
\quad \times \frac{(T-n k)^{1-\alpha}}{\Gamma(2-\alpha)}-\frac{\Gamma(1+\alpha)}{2} \\
\quad \times \sigma^{2} m^{2}\left(V_{m+1}^{n}-2 V_{m}^{n}+V_{m-1}^{n}\right)=0,
\end{gathered}
$$

where the local truncation error is $O\left(k+h^{2}\right)$.

From (11), we get the following equality when $n=1$ :

$$
\begin{aligned}
& k^{(\alpha-1)}(T-k)^{(1-\alpha)} \varrho_{\alpha, k}\left(V_{m}^{1}-V_{m}^{0}\right) \\
& +\left(r V_{m}^{1}-r m \frac{V_{m+1}^{1}-V_{m-1}^{1}}{2}\right) \\
& \quad \times \frac{(T-k)^{1-\alpha}}{\Gamma(2-\alpha)}-\frac{\Gamma(1+\alpha)}{2} \\
& \quad \times \sigma^{2} m^{2}\left(V_{m+1}^{1}-2 V_{m}^{1}+V_{m-1}^{1}\right)=0 .
\end{aligned}
$$

After combining like terms, we can derive the following equation:

$$
\begin{gathered}
\left(\frac{r m(T-k)^{1-\alpha}}{2 \Gamma(2-\alpha)}-\frac{\Gamma(1+\alpha)}{2} \sigma^{2} m^{2}\right) V_{m-1}^{1} \\
+\left(k^{(\alpha-1)}(T-k)^{(1-\alpha)} \varrho_{\alpha, k}+\frac{r(T-k)^{1-\alpha}}{\Gamma(2-\alpha)}\right. \\
\left.+\Gamma(1+\alpha) \sigma^{2} m^{2}\right) V_{m}^{1}
\end{gathered}
$$

$$
\begin{aligned}
& -\left(\frac{\Gamma(1+\alpha)}{2} \sigma^{2} m^{2}+\frac{r m(T-k)^{1-\alpha}}{2 \Gamma(2-\alpha)}\right) V_{m+1}^{1} \\
= & k^{(\alpha-1)}(T-k)^{(1-\alpha)} \varrho_{\alpha, k} V_{m}^{0}, \\
& m=1,2, \ldots, M-1 .
\end{aligned}
$$

For $n \geq 2$, we obtain

$$
\begin{gathered}
\left(\frac{r m(T-n k)^{1-\alpha}}{2 \Gamma(2-\alpha)}-\frac{\Gamma(1+\alpha)}{2} \sigma^{2} m^{2}\right) V_{m-1}^{n} \\
+\left((n k)^{(\alpha-1)}(T-n k)^{(1-\alpha)} \varrho_{\alpha, k}\right. \\
\left.\quad+\frac{r(T-n k)^{1-\alpha}}{\Gamma(2-\alpha)}+\Gamma(1+\alpha) \sigma^{2} m^{2}\right) V_{m}^{n} \\
\quad-\left(\frac{\Gamma(1+\alpha)}{2} \sigma^{2} m^{2}+\frac{r m(T-n k)^{1-\alpha}}{2 \Gamma(2-\alpha)}\right) V_{m+1}^{n} \\
=(n k)^{(\alpha-1)}(T-n k)^{(1-\alpha)} \varrho_{\alpha, k} \\
\times\left(V_{m}^{n-1}-\sum_{j=2}^{n} \omega_{j}^{(\alpha)}\left(V_{m}^{n-j+1}-V_{m}^{n-j}\right)\right), \\
m=1,2, \ldots, M-1 .
\end{gathered}
$$

If the minimum limit of variable $S$ is $S_{\min }$, the corresponding implicit difference approximation can be derived by replacing $m$ of the above results (13) and (14) with $\left(S_{\min } / h\right)+$ $m$.

From the terminal and boundary conditions of the European put option, we can get

$$
\begin{aligned}
V_{m}^{0} & =\max (K-m h, 0), \quad m=0, \ldots, M, \\
V_{0}^{n} & =K \exp (-r n k), \quad n=1, \ldots, N, \\
V_{M}^{n} & =0, \quad n=1, \ldots, N .
\end{aligned}
$$

In the case of American put option, we perform the above procedure. At the same time, we should check for the possibility of early exercise after computing $V_{m}^{n}$ and set

$$
V_{m}^{n}=\max \left(V_{m}^{n}, K-m h\right), \quad \text { for } n=1, \ldots, N
$$

Under this implicit scheme, we do not directly apply (16) at each step but use SOR method to complete this procedure, which was suggested in [16].

\section{Stability and Convergence}

In this section, we will analyze the stability and convergence of implicit finite difference scheme (13) and (14) using Fourier 
analysis involved in [39-41]. For that, (13) and (14) are rewritten as

$$
\begin{gathered}
a_{m, 1} V_{m-1}^{1}+b_{m, 1} V_{m}^{1}+c_{m, 1} V_{m+1}^{1}=V_{m}^{0} \\
a_{m, n} V_{m-1}^{n}+b_{m, n} V_{m}^{n}+c_{m, n} V_{m+1}^{n} \\
=\left(1-\omega_{2}^{(\alpha)}\right) V_{m}^{n-1}-\sum_{j=2}^{n-1}\left(\omega_{j+1}^{(\alpha)}-\omega_{j}^{(\alpha)}\right) V_{m}^{n-j} \\
+\omega_{n}^{(\alpha)} V_{m}^{0}
\end{gathered}
$$

where

$$
\begin{aligned}
a_{m, n}= & (n k)^{(1-\alpha)}(T-n k)^{(\alpha-1)} \varrho_{\alpha, k}^{-1} \\
& \times\left(\frac{r m(T-n k)^{1-\alpha}}{2 \Gamma(2-\alpha)}-\frac{\Gamma(1+\alpha)}{2} \sigma^{2} m^{2}\right), \\
b_{m, n}= & 1+(n k)^{(1-\alpha)}(T-n k)^{(\alpha-1)} \varrho_{\alpha, k}^{-1} \\
& \times\left(\frac{r(T-n k)^{1-\alpha}}{\Gamma(2-\alpha)}+\Gamma(1+\alpha) \sigma^{2} m^{2}\right), \\
c_{m, n}= & -(n k)^{(1-\alpha)}(T-n k)^{(\alpha-1)} \varrho_{\alpha, k}^{-1} \\
& \times\left(\frac{\Gamma(1+\alpha)}{2} \sigma^{2} m^{2}+\frac{r m(T-n k)^{1-\alpha}}{2 \Gamma(2-\alpha)}\right) .
\end{aligned}
$$

3.1. Stability Analysis. If $U_{m}^{n}$ is another approximate solution of (13) and (14), we define the round-off error $\varepsilon_{m}^{n}=V_{m}^{n}-U_{m}^{n}$ which satisfies the following equations according to (17):

$$
\begin{gathered}
a_{m, 1} \varepsilon_{m-1}^{1}+b_{m, 1} \varepsilon_{m}^{1}+c_{m, 1} \varepsilon_{m+1}^{1}=\varepsilon_{m}^{0}, \\
a_{m, n} \varepsilon_{m-1}^{n}+b_{m, n} \varepsilon_{m}^{n}+c_{m, n} \varepsilon_{m+1}^{n} \\
=\left(1-\omega_{2}^{(\alpha)}\right) \varepsilon_{m}^{n-1} \\
\quad-\sum_{j=2}^{n-1}\left(\omega_{j+1}^{(\alpha)}-\omega_{j}^{(\alpha)}\right) \varepsilon_{m}^{n-j}+\omega_{n}^{(\alpha)} \varepsilon_{m}^{0}, \\
n=2, \ldots, N, \quad m=1,2, \ldots, M-1, \\
\varepsilon_{0}^{n}=\varepsilon_{M}^{n}=0, \quad n=0, \ldots, N .
\end{gathered}
$$

Now, we introduce the grid function in [39]:

$\varepsilon^{n}(S)=\left\{\begin{array}{c}\varepsilon_{m}^{n}, \quad \text { when } S_{m}-\frac{h}{2}<S \leq S_{m}+\frac{h}{2} \\ m=1, \ldots, M-1, \\ 0, \quad \text { when } 0 \leq S \leq \frac{h}{2} \text { or } S_{\max }-\frac{h}{2}<S \leq S_{\max } .\end{array}\right.$

Recall the result of [39]; defining

$$
\begin{gathered}
\varepsilon^{n}=\left[\varepsilon_{1}^{n}, \varepsilon_{2}^{n}, \ldots, \varepsilon_{M-1}^{n}\right]^{T} \\
\left\|\varepsilon^{n}\right\|_{2}=\left(\sum_{m=1}^{M-1} h\left|\varepsilon_{m}^{n}\right|^{2}\right)^{1 / 2}=\left(\int_{0}^{S_{\max }}\left|\varepsilon^{n}(S)\right|^{2} d S\right)^{1 / 2}
\end{gathered}
$$

can get

$$
\left\|\varepsilon^{n}\right\|_{2}^{2}=\sum_{l=-\infty}^{+\infty}\left|\xi_{n}(l)\right|^{2},
$$

where $\xi_{n}(l)=\left(1 / S_{\max }\right) \int_{0}^{S_{\max }} \varepsilon^{n}(S) \exp \left(-I 2 \pi l S / S_{\max }\right) d S$.

The solution of (19) and (20) is supposed to have the following form:

$$
\varepsilon_{m}^{n}=\xi_{n} \exp (I \mu m h),
$$

where $\mu=2 \pi l / S_{\max }, I=\sqrt{-1}$.

Substituting the above expression into (19), we get

$$
\begin{gathered}
a_{m, 1} \xi_{1} \exp (I \mu(m-1) h)+b_{m, 1} \xi_{1} \exp (I \mu m h) \\
+c_{m, 1} \xi_{1} \exp (I \mu(m+1) h)=\xi_{0} \exp (I \mu m h), \\
a_{m, n} \xi_{n} \exp (I \mu(m-1) h)+b_{m, n} \xi_{n} \exp (I \mu m h) \\
+c_{m, n} \xi_{n} \exp (I \mu(m+1) h) \\
=\left(1-\omega_{2}^{(\alpha)}\right) \xi_{n-1} \exp (I \mu m h) \\
\quad-\sum_{j=2}^{n-1}\left(\omega_{j+1}^{(\alpha)}-\omega_{j}^{(\alpha)}\right) \xi_{n-j} \exp (I \mu m h) \\
+\omega_{n}^{(\alpha)} \xi_{0} \exp (I \mu m h) .
\end{gathered}
$$

Simplifying (25), we obtain

$$
\begin{gathered}
\vartheta_{m, 1} \xi_{1}=\xi_{0} \\
\vartheta_{m, n} \xi_{n}=\left(1-\omega_{2}^{(\alpha)}\right) \xi_{n-1}-\sum_{j=2}^{n-1}\left(\omega_{j+1}^{(\alpha)}-\omega_{j}^{(\alpha)}\right) \xi_{n-j}+\omega_{n}^{(\alpha)} \xi_{0}
\end{gathered}
$$

where $\vartheta_{m, n}=1+(n k)^{(1-\alpha)}(T-n k)^{(\alpha-1)} \varrho_{\alpha, k}^{-1}\left[\left(r(T-n k)^{1-\alpha} / \Gamma(2-\right.\right.$ $\left.\alpha))(1-\operatorname{Im} \sin (\mu h))+\Gamma(1+\alpha) \sigma^{2} m^{2}(1-\cos (\mu h))\right]$.

Proposition 1. If $\xi_{n}$ is a solution of (26), then $\left|\xi_{n}\right| \leq\left|\xi_{0}\right|$.

Proof. For $n=1$, the first equality of (26) gives $\left|\xi_{1}\right|=(1 /$ $\left.\left|\vartheta_{m, 1}\right|\right)\left|\xi_{0}\right| \leq\left|\xi_{0}\right|$. If

$$
\left|\xi_{n-1}\right| \leq\left|\xi_{0}\right|
$$

then using the second equality of (26), we obtain

$$
\begin{aligned}
\left|\xi_{n}\right| & \leq \frac{1-\omega_{2}^{(\alpha)}-\left(\omega_{n}^{(\alpha)}-\omega_{2}^{(\alpha)}\right)+\omega_{n}^{(\alpha)}}{\left|\vartheta_{m, n}\right|}\left|\xi_{0}\right| \\
& =\frac{1}{\left|\vartheta_{m, n}\right|}\left|\xi_{0}\right| \leq\left|\xi_{0}\right| .
\end{aligned}
$$

According to (13) and (14), we can obtain the following conclusion using Proposition 1 and equality (23).

Theorem 2. The difference scheme (13) and (14) is unconditionally stable. 
3.2. Convergence Analysis. Suppose that $V\left(S_{i}, t_{k}\right)$ is an exact solution of (7) at grid point $\left(S_{i}, t_{k}\right)$ and $V_{k}^{i}$ is the difference solution of (13) and (14); we define the error $\epsilon_{j}^{k}=V\left(S_{i}, t_{k}\right)-V_{k}^{i}$ which satisfies the following equation according to (17):

$$
\begin{gathered}
a_{m, 1} \epsilon_{m-1}^{1}+b_{m, 1} \epsilon_{m}^{1}+c_{m, 1} \epsilon_{m+1}^{1}=k^{\alpha} R_{m}^{1}, \\
a_{m, n} \epsilon_{m-1}^{n}+b_{m, n} \epsilon_{m}^{n}+c_{m, n} \epsilon_{m+1}^{n} \\
=\left(1-\omega_{2}^{(\alpha)}\right) \epsilon_{m}^{n-1} \\
\quad-\sum_{j=2}^{n-1}\left(\omega_{j+1}^{(\alpha)}-\omega_{j}^{(\alpha)}\right) \epsilon_{m}^{n-j}+k^{\alpha} R_{m}^{n}, \\
n=2, \ldots, N, \quad m=1,2, \ldots, M-1, \\
\epsilon_{0}^{n}=\epsilon_{M}^{n}=0, \quad n=1, \ldots, N, \\
\epsilon_{m}^{0}=0, \quad m=0, \ldots, M,
\end{gathered}
$$

where $\left|R_{m}^{n}\right| \leq C_{1}\left(k+h^{2}\right)(n=1,2, \ldots, N)$ and $C_{1}$ is a positive constant.

For completing the proof of convergence, we recall several results below which came from [39].

Similar to the stability analysis, [39] constructed the following grid function:

$$
\begin{gathered}
\epsilon^{n}(S)=\left\{\begin{array}{c}
\epsilon_{m}^{n}, \quad \text { when } S_{m}-\frac{h}{2}<S \leq S_{m}+\frac{h}{2}, \\
m=1, \ldots, M-1, \\
0, \quad \text { when } 0 \leq S \leq \frac{h}{2} \text { or } S_{\max }-\frac{h}{2}<S \leq S_{\max },
\end{array}\right. \\
R^{n}(S)=\left\{\begin{array}{rc}
R_{m}^{n}, & \text { when } S_{m}-\frac{h}{2}<S \leq S_{m}+\frac{h}{2}, \\
m=1, \ldots, M-1, & \\
0, & \text { when } 0 \leq S \leq \frac{h}{2} \text { or } S_{\max }-\frac{h}{2}<S \leq S_{\max } .
\end{array}\right.
\end{gathered}
$$

Defining

$$
\epsilon^{n}=\left[\epsilon_{1}^{n}, \epsilon_{2}^{n}, \ldots, \epsilon_{M-1}^{n}\right]^{T}, \quad R^{n}=\left[R_{1}^{n}, R_{2}^{n}, \ldots, R_{M-1}^{n}\right]^{T}
$$

with the norms

$$
\begin{aligned}
\left\|\epsilon^{n}\right\|_{2} & =\left(\sum_{m=1}^{M-1} h\left|\epsilon_{m}^{n}\right|^{2}\right)^{1 / 2}=\left(\int_{0}^{S_{\max }}\left|\epsilon^{n}(S)\right|^{2} d S\right)^{1 / 2}, \\
\left\|R^{n}\right\|_{2} & =\left(\sum_{m=1}^{M-1} h\left|R_{m}^{n}\right|^{2}\right)^{1 / 2}=\left(\int_{0}^{S_{\max }}\left|R^{n}(S)\right|^{2} d S\right)^{1 / 2}
\end{aligned}
$$

can obtain

$$
\begin{aligned}
\left\|\epsilon^{n}\right\|_{2}^{2} & =\sum_{l=-\infty}^{+\infty}\left|\zeta_{n}(l)\right|^{2}, \\
\left\|R^{n}\right\|_{2}^{2} & =\sum_{l=-\infty}^{+\infty}\left|\eta_{n}(l)\right|^{2},
\end{aligned}
$$

where

$$
\begin{aligned}
& \zeta_{n}(l)=\frac{1}{S_{\max }} \int_{0}^{S_{\max }} \epsilon^{n}(S) \exp \left(-\frac{I 2 \pi l S}{S_{\max }}\right) d S, \\
& \eta_{n}(l)=\frac{1}{S_{\max }} \int_{0}^{S_{\max }} R^{n}(S) \exp \left(-\frac{I 2 \pi l S}{S_{\max }}\right) d S .
\end{aligned}
$$

We can let

$$
\begin{aligned}
\epsilon_{m}^{n} & =\zeta_{n} \exp (I \mu m h), \\
R_{m}^{n} & =\eta_{n} \exp (I \mu m h),
\end{aligned}
$$

where $\mu=2 \pi l / S_{\max }, I=\sqrt{-1}$.

Substituting the above expressions into (29) and simplifying them can get

$$
\begin{gathered}
\vartheta_{m, 1} \zeta_{1}=k^{\alpha} \eta_{1}, \\
\vartheta_{m, n} \zeta_{n}=\left(1-\omega_{2}^{(\alpha)}\right) \zeta_{n-1}-\sum_{j=2}^{n-1}\left(\omega_{j+1}^{(\alpha)}-\omega_{j}^{(\alpha)}\right) \zeta_{n-j}+k^{\alpha} \eta_{n},
\end{gathered}
$$

where $\vartheta_{m, n}=1+(n k)^{(1-\alpha)}(T-n k)^{(\alpha-1)} \varrho_{\alpha, k}^{-1}\left(\left[\left(r(T-n k)^{1-\alpha} / \Gamma(2-\right.\right.\right.$ $\left.\alpha))(1-\operatorname{Im} \sin (\mu h))+\Gamma(1+\alpha) \sigma^{2} m^{2}(1-\cos (\mu h))\right]$.

Proposition 3. There exists a positive constant $C_{2}$, so that $\left|\zeta_{n}\right| \leq C_{2} n k^{\alpha}\left|\eta_{1}\right|$

Proof. From [39], we know

$$
\begin{gathered}
\left\|R^{n}\right\|_{2} \leq C_{1} \sqrt{S_{\max }}\left(k+h^{2}\right) \quad(n=1,2, \ldots, N), \\
\left|\eta_{n}\right| \leq C_{2}\left|\eta_{1}\right| \quad(n=1,2, \ldots, N) .
\end{gathered}
$$

Then the first equality of (37) tells us that

$$
\left|\zeta_{1}\right| \leq \frac{1}{\left|\vartheta_{m, n}\right|} k^{\alpha}\left|\eta_{1}\right| \leq C_{2} k^{\alpha}\left|\eta_{1}\right| .
$$

If we let

$$
\left|\zeta_{n-1}\right| \leq C_{2}(n-1) k^{\alpha}\left|\eta_{1}\right|,
$$

using the second equality of (37), we can obtain

$$
\left|\zeta_{n}\right| \leq \frac{1}{\left|\vartheta_{m, n}\right|} \mid\left(1-\omega_{2}^{(\alpha)}\right)(n-1)
$$

$$
\begin{aligned}
& \quad+\sum_{j=2}^{n-1}\left(\omega_{j}^{(\alpha)}-\omega_{j+1}^{(\alpha)}\right)(n-j)+1\left|C_{2} k^{\alpha}\right| \eta_{1} \mid \\
& \leq \frac{1}{\left|\vartheta_{m, n}\right|} \mid\left(1-\omega_{2}^{(\alpha)}\right)(n-1) \\
& \quad+\sum_{j=2}^{n-1}\left(\omega_{j}^{(\alpha)}-\omega_{j+1}^{(\alpha)}\right)(n-1)+1\left|C_{2} k^{\alpha}\right| \eta_{1} \mid \\
& \leq \frac{1}{\left|\vartheta_{m, n}\right|}\left|\left(1-\omega_{n}^{(\alpha)}\right)(n-1)+1\right| C_{2} k^{\alpha}\left|\eta_{1}\right| \\
& \leq n C_{2} k^{\alpha}\left|\eta_{1}\right| .
\end{aligned}
$$




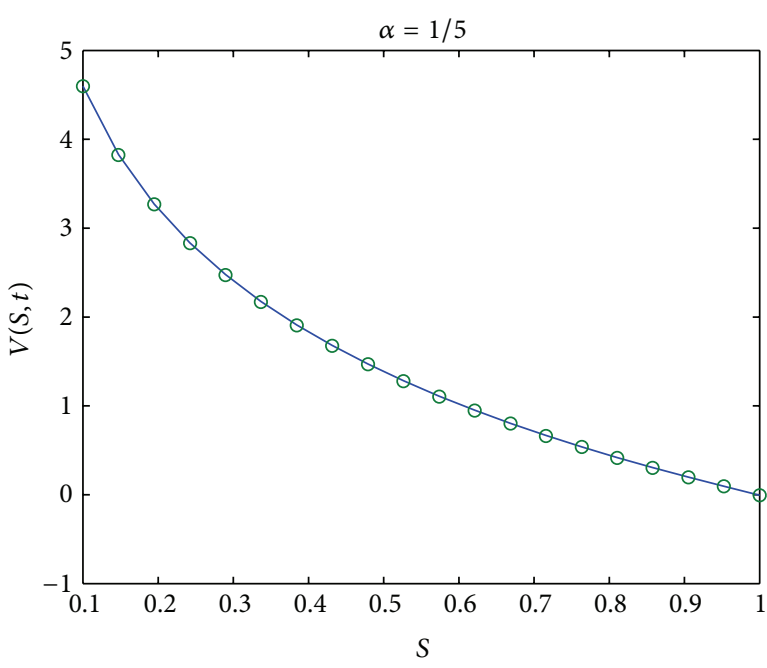

(a)

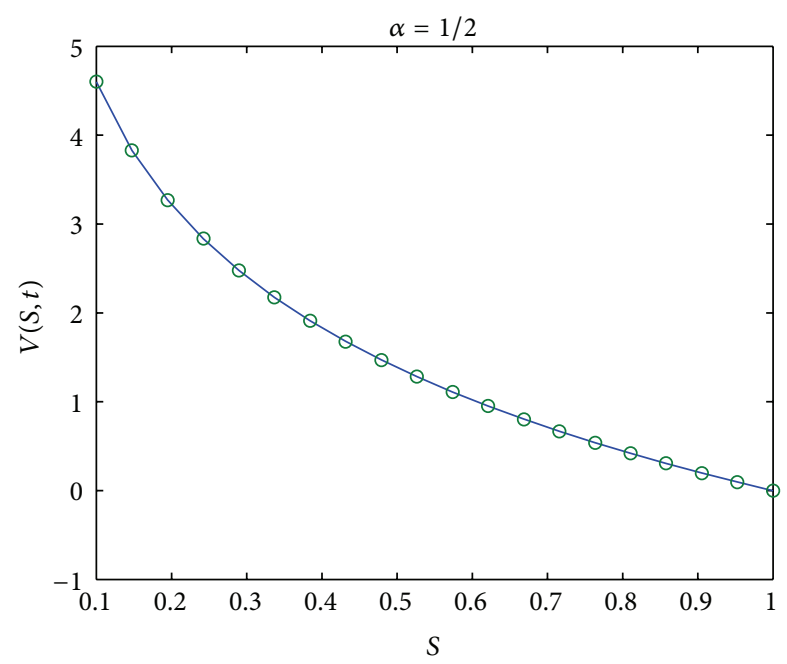

(b)

FIGURE 1: Solution curve with $t=0.5$. Solid line: numerical solution; Soft dot: analytical solution.

According to equalities (34) and inequality (38), we can obtain the following conclusion using Proposition 3.

Theorem 4. The implicit difference scheme (13) and (14) is $L_{2}{ }^{-}$ convergent.

\section{Computational Examples}

Option pricing model based on the time-fractional differential equation (1) is studied. The implicit difference scheme of (7) is given in Section 2. From the finite difference forms, it is clear that (13) and (14) are just the implicit difference scheme of the classical Black-Scholes equation if we let $\alpha=1$. This known implicit difference results are given in $[16,17,36]$. In this section, we focus mainly on investigating the results with $0<\alpha<1$. To explain the stability and convergence of the implicit numerical schemes, we firstly take Example 1 with the given terminal and boundary condition as an example.

Example 1. Consider (1) that is subject to the following conditions:

$$
\begin{gathered}
V(S, T)=V(S, T)=\sigma^{2}\left(2 T-T^{\alpha}\right)-2 \ln (S) \\
V\left(S_{\min }, t\right)=\exp (-r(T-t))\left(\sigma^{2}\left(2 t-t^{\alpha}\right)-2 \ln \left(S_{\min }\right)\right), \\
V\left(S_{\max }, t\right)=\exp (-r(T-t))\left(\sigma^{2}\left(2 t-t^{\alpha}\right)-2 \ln \left(S_{\max }\right)\right) .
\end{gathered}
$$

Under the condition $r=\sigma^{2}$, (1) has an analytical solution

$$
V(S, t)=\exp (-r(T-t))\left(\sigma^{2}\left(2 t-t^{\alpha}\right)-2 \ln (S)\right) .
$$

Figure 1 shows analytical solution and numerical solution obtained by the implicit difference method at time $t=0.5$ when $r=0.01, \sigma=0.1, T=1, S_{\min }=0.1, S_{\max }=1, M=$ 19 , and $N=10$. Numerical solution compares well with analytical solution, which proves that the implicit scheme is stable. Under the same parameters, Figure 2 gives the absolute error between numerical solutions and analytical solutions, which illustrates that the numerical results are convergent.

Example 2. European put option pricing model is based on (1) and the condition (4) under the following parameters:

$$
\begin{gathered}
K=50, \quad r=0.01, \quad \sigma=0.3, \quad T=1, \\
S_{\max }=100, \quad M=20, \quad N=10 .
\end{gathered}
$$

Through coding, the European put option values with $\alpha=$ $1,1 / 2,5 / 7,9 / 10$ are plotted in Figure 3 at $t=0.1 / 0.5 / 1$.

Example 3. Consider American put option pricing model. The following parameters are selected for the present study:

$$
\begin{gathered}
K=60, \quad r=0.01, \quad \sigma=0.4, \quad T=1, \\
S_{\max }=120, \quad M=30, \quad N=10 .
\end{gathered}
$$

Figure 4 indicates a price comparison of the American put option at $t=0.2 / 0.6 / 1$ (relaxation parameter and tolerance parameter are 1.2 and 0.001 in the applied process of SOR method).

Using Examples 1, 2, and 3, we examine the implementation of the implicit finite difference method for the fractional partial differential equation system. According to Figures 1 and 2 in Example 1, we can confirm that the implicit numerical scheme is stable and convergent. From Figures 3 and 4, we can see that the numerical scheme is very effective. Figures 3 and 4 present numerical simulation of the price of the European and American put options when the order of the time-fractional derivative takes different values. Their visible shapes and development trend are similar to the classical put option pricing model based on the standard Black-Scholes equation, which illustrates the essential characteristics of the European and American put options. For making the figures 


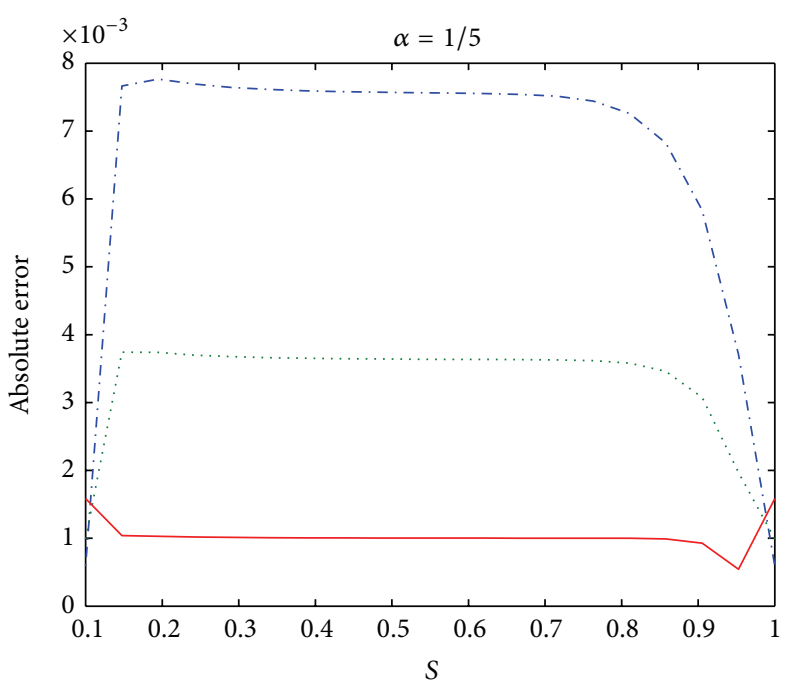

(a)

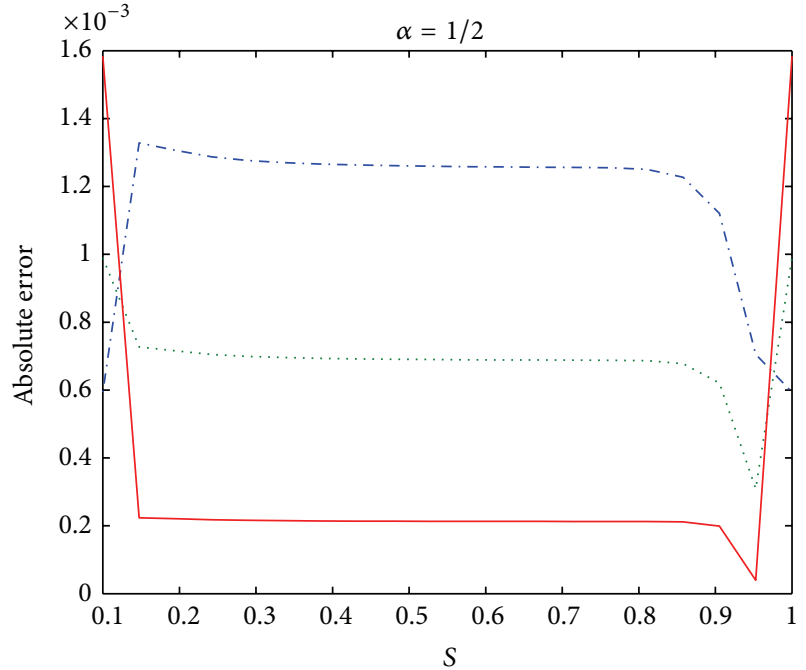

(b)

FIGURE 2: Absolute error between numerical solution and analytical solution. dash-dotted line: $t=0.3$; dotted line: $t=0.5$; solid line: $t=0.8$.

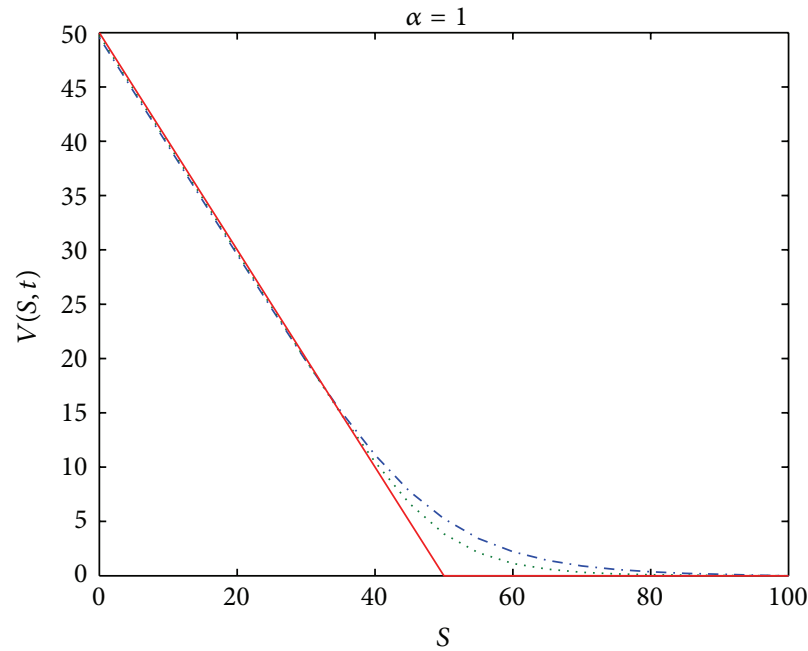

(a)

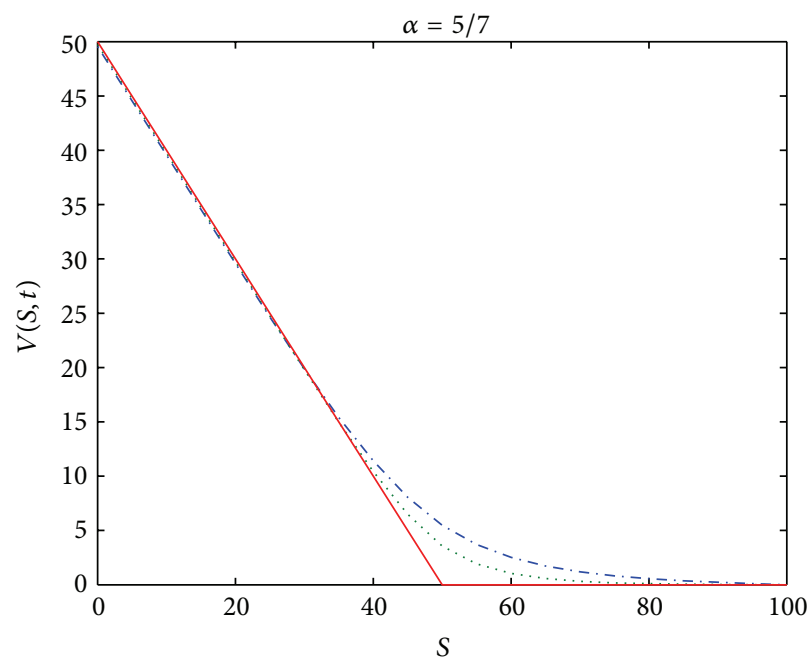

(c)

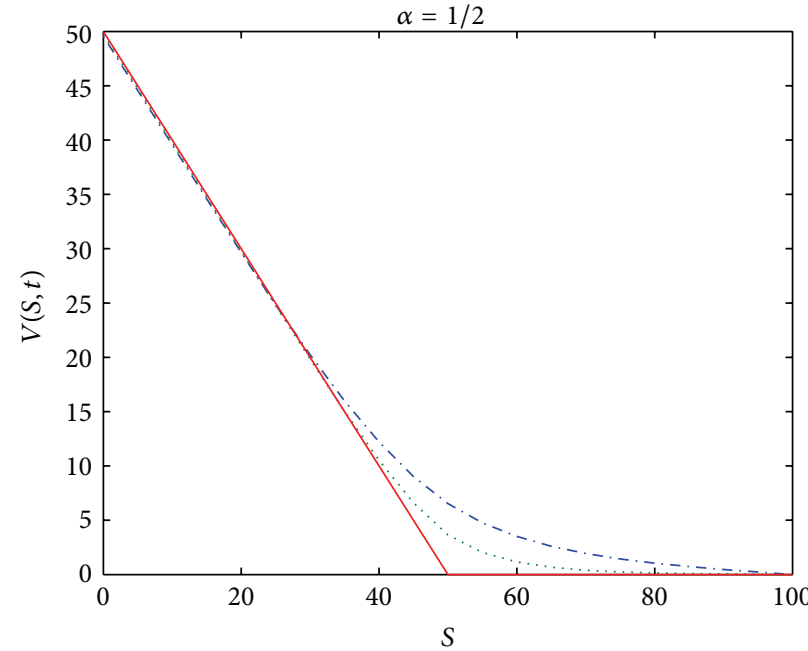

(b)

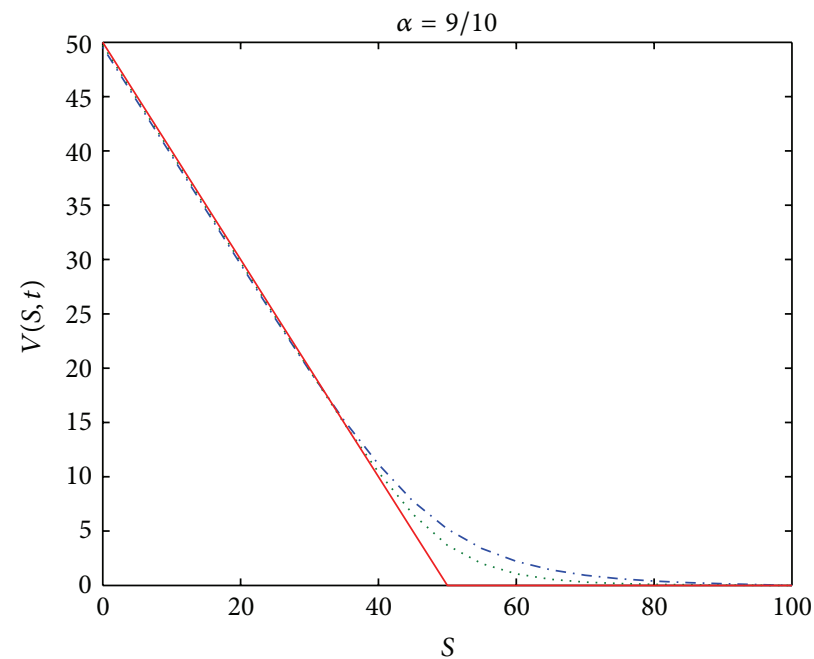

(d)

FIGURE 3: European put option. dash-dotted line: $t=0.1$; dotted line: $t=0.5$; solid line: $t=1$. 


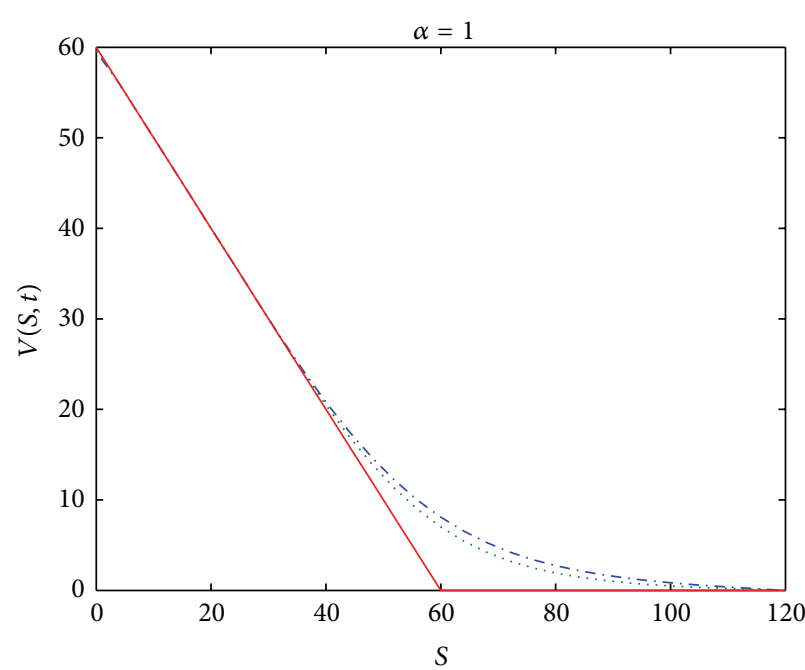

(a)

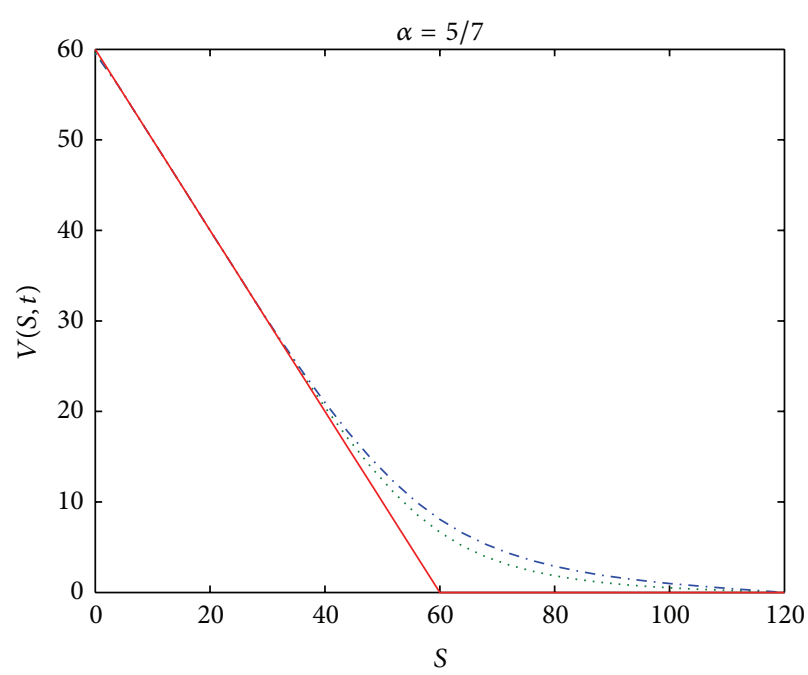

(c)

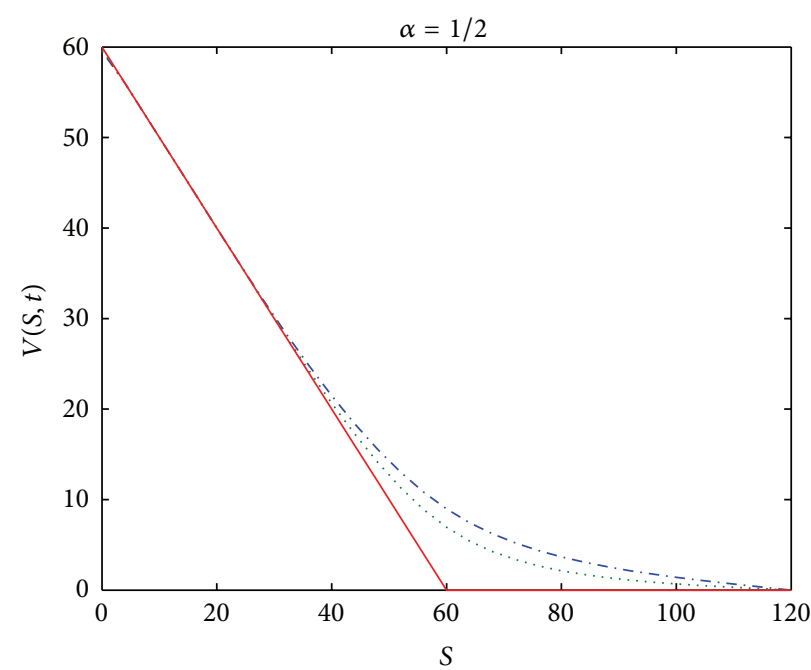

(b)

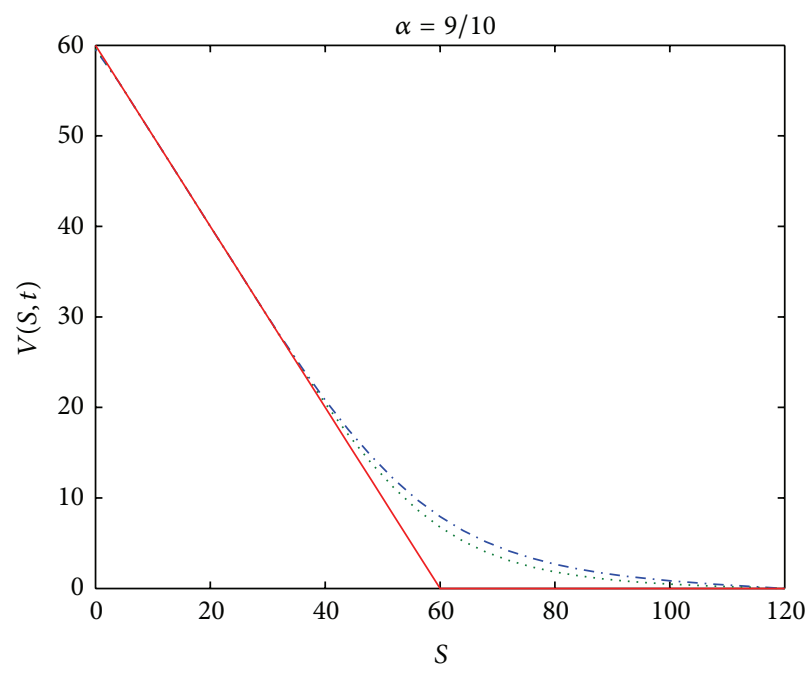

(d)

FIgURE 4: American put option. dash-dotted line: $t=0.2$; dotted line: $t=0.6$; solid line: $t=1$.

clear, we choose the small values of $M$ and $N$ and get the similar conclusions if largening the number of steps in time and space. As a generalization of the standard models, these fractional Black-Scholes models are powerful and will be of great interest to researchers in further work.

\section{Conclusions}

In this work, the finite difference method is employed to solve the time-fractional Black-Scholes equation together with the conditions satisfied by the standard put options. Application of the fractional differential equation to the pricing theory of option is in its beginning stage and needs more further work. This fractional model mentioned in this paper can model the price of other financial derivatives like warrant, swaps, and so on. The successful application of the finite difference method proves that this technique is effective and requires less computational work to solve fractional partial differential equation.

\section{Acknowledgments}

The authors would like to express their gratitude to reviewers for the careful reading of the paper and for their constructive comments which greatly improve the quality of this paper. The work is partially supported by the National Natural Science Foundation of China (no. 71171035, no. 71273044, and no. 71271045).

\section{References}

[1] R. C. Merton, "On the pricing of corporate debt: the risk structure of interest rates," Journal of Finance, vol. 29, pp. 449470, 1974.

[2] R. C. Merton, "Option pricing when underlying stock returns are discontinuous," Journal of Financial Economics, vol. 3, no. 1-2, pp. 125-144, 1976.

[3] J. C. Hull and A. D. White, "The pricing of options on assets with stochastic volatilities," Journal of Finance, vol. 42, pp. 281-300, 1987. 
[4] M. H. A. Davis, V. G. Panas, and T. Zariphopoulou, "European option pricing with transaction costs," SIAM Journal on Control and Optimization, vol. 31, no. 2, pp. 470-493, 1993.

[5] G. Barles and H. M. Soner, "Option pricing with transaction costs and a nonlinear Black-Scholes equation," Finance and Stochastics, vol. 2, no. 4, pp. 369-397, 1998.

[6] T. Björk and H. Hult, "A note on Wick products and the fractional Black-Scholes model," Finance and Stochastics, vol. 9, no. 2, pp. 197-209, 2005.

[7] X.-T. Wang, "Scaling and long-range dependence in option pricing I: Pricing European option with transaction costs under the fractional Black-Scholes model," Physica A, vol. 389, no. 3, pp. 438-444, 2010.

[8] J.-R. Liang, J. Wang, W.-J. Zhang, W.-Y. Qiu, and F.-Y. Ren, "Option pricing of a bi-fractional Black-Merton-Scholes model with the Hurst exponent $H$ in [1/2,1]," Applied Mathematics Letters, vol. 23, no. 8, pp. 859-863, 2010.

[9] J. Wang, J.-R. Liang, L.-J. Lv, W.-Y. Qiu, and F.-Y. Ren, “Continuous time Black-Scholes equation with transaction costs in subdiffusive fractional Brownian motion regime," Physica A, vol. 391, no. 3, pp. 750-759, 2012.

[10] W. Wyss, "The fractional Black-Scholes equation," Fractional Calculus \& Applied Analysis for Theory and Applications, vol. 3, no. 1, pp. 51-61, 2000.

[11] Á. Cartea and D. del-Castillo-Negrete, "Fractional diffusion models of option prices in markets with jumps," Physica A, vol. 374, no. 2, pp. 749-763, 2007.

[12] G. Jumarie, "Stock exchange fractional dynamics defined as fractional exponential growth driven by (usual) Gaussian white noise. Application to fractional Black-Scholes equations," Insurance: Mathematics \& Economics, vol. 42, no. 1, pp. 271-287, 2008.

[13] G. Jumarie, "Derivation and solutions of some fractional BlackScholes equations in coarse-grained space and time. Application to Merton's optimal portfolio," Computers \& Mathematics with Applications, vol. 59, no. 3, pp. 1142-1164, 2010.

[14] G. Jumarie, "Modified Riemann-Liouville derivative and fractional Taylor series of nondifferentiable functions further results," Computers \& Mathematics with Applications, vol. 51, no. 9-10, pp. 1367-1376, 2006.

[15] G. Jumarie, "Cauchy's integral formula via the modified Riemann-Liouville derivative for analytic functions of fractional order," Applied Mathematics Letters of Rapid Publication, vol. 23, no. 12, pp. 1444-1450, 2010.

[16] P. Brandimarte, Numerical Methods in Finance and Economics, John Wiley \& Sons, Hoboken, NJ, USA, 2006.

[17] S. Sukha, "Advanced mathematics of finance honours project: finite-difference methods for pricing the American put option," 2001.

[18] E. Sousa, "A second order explicit finite difference method for the fractional advection diffusion equation," Computers \& Mathematics with Applications, vol. 64, no. 10, pp. 3141-3152, 2012.

[19] A. Mohebbi and M. Abbaszadeh, "Compact finite difference scheme for the solution of time fractional advection-dispersion equation," Numerical Algorithms, 2012.

[20] M. M. Meerschaert and C. Tadjeran, "Finite difference approximations for fractional advection-dispersion flow equations," Journal of Computational and Applied Mathematics, vol. 172, no. 1, pp. 65-77, 2004.

[21] F. Liu, P. Zhuang, and K. Burrage, "Numerical methods and analysis for a class of fractional advection-dispersion models," Computers \& Mathematics with Applications, vol. 64, no. 10, pp. 2990-3007, 2012.
[22] F. Liu, P. Zhuang, V. Anh, I. Turner, and K. Burrage, "Stability and convergence of the difference methods for the space-time fractional advection-diffusion equation," Applied Mathematics and Computation, vol. 191, no. 1, pp. 12-20, 2007.

[23] P. Zhuang, Y. T. Gu, F. Liu, I. Turner, and P. K. D. V. Yarlagadda, "Time-dependent fractional advection-diffusion equations by an implicit MLS meshless method," International Journal for Numerical Methods in Engineering, vol. 88, no. 13, pp. 1346-1362, 2011.

[24] A. M. A. El-Sayed, S. H. Behiry, and W. E. Raslan, "Adomian's decomposition method for solving an intermediate fractional advection-dispersion equation," Computers \& Mathematics with Applications, vol. 59, no. 5, pp. 1759-1765, 2010.

[25] A. Golbabai and K. Sayevand, "Analytical modelling of fractional advection-dispersion equation defined in a bounded space domain," Mathematical and Computer Modelling, vol. 53, no. 9-10, pp. 1708-1718, 2011.

[26] G. H. Zheng and T. Wei, "Spectral regularization method for a Cauchy problem of the time fractional advection-dispersion equation," Journal of Computational and Applied Mathematics, vol. 233, no. 10, pp. 2631-2640, 2010.

[27] S. Liao, "An optimal homotopy-analysis approach for strongly nonlinear differential equations," Communications in Nonlinear Science and Numerical Simulation, vol. 15, no. 8, pp. 2003-2016, 2010.

[28] A.-M. Wazwaz, "New solitary wave solutions to the KuramotoSivashinsky and the Kawahara equations," Applied Mathematics and Computation, vol. 182, no. 2, pp. 1642-1650, 2006.

[29] Z. Yan, "New explicit travelling wave solutions for two new integrable coupled nonlinear evolution equations," Physics Letters A, vol. 292, no. 1-2, pp. 100-106, 2001.

[30] Q. Wang, Y. Chen, and H. Zhang, "A new Riccati equation rational expansion method and its application to $(2+1)$ dimensional Burgers equation," Chaos, Solitons \& Fractals, vol. 25, no. 5, pp. 1019-1028, 2005.

[31] Z. Odibat, S. Momani, and H. Xu, "A reliable algorithm of homotopy analysis method for solving nonlinear fractional differential equations," Applied Mathematical Modelling, vol. 34, no. 3, pp. 593-600, 2010.

[32] S. Abbasbandy, M. Ashtiani, and E. Babolian, "Analytic solution of the Sharma-Tasso-Olver equation by homotopy analysis method," Zeitschrift für Naturforschung-Section A, vol. 65, no. 4, pp. 285-290, 2010.

[33] E. Fan, "Extended tanh-function method and its applications to nonlinear equations," Physics Letters A, vol. 277, no. 4-5, pp. 212-218, 2000.

[34] S.-P. Zhu and G.-H. Lian, "A closed-form exact solution for pricing variance swaps with stochastic volatility," Mathematical Finance, vol. 21, no. 2, pp. 233-256, 2011.

[35] L. V. Ballestra and C. Sgarra, "The evaluation of American options in a stochastic volatility model with jumps: an efficient finite element approach," Computers \& Mathematics with Applications, vol. 60, no. 6, pp. 1571-1590, 2010.

[36] L. S. Jiang, Mathematical Modeling and Methods of Option Pricing, Higher Education press, Beijing, China, 2003.

[37] J. Ankudinova and M. Ehrhardt, "On the numerical solution of nonlinear Black-Scholes equations," Computers \& Mathematics with Applications, vol. 56, no. 3, pp. 799-812, 2008.

[38] D. A. Murio, "Implicit finite difference approximation for time fractional diffusion equations," Computers \& Mathematics with Applications, vol. 56, no. 4, pp. 1138-1145, 2008.

[39] C.-M. Chen, F. Liu, and K. Burrage, "Finite difference methods and a Fourier analysis for the fractional reaction-subdiffusion 
equation," Applied Mathematics and Computation, vol. 198, no. 2, pp. 754-769, 2008.

[40] H. F. Ding and Y. X. Zhang, "Notes on implicit finite difference approximation for timefractional diffusion equations," Computers \& Mathematics with Applications, vol. 61, no. 9, pp. 29242928, 2011.

[41] D. A. Murio, "Implicit finite difference approximationfor time fractional diffusion equations," Computers \& Mathematics with Applications, vol. 56, no. 4, pp. 1138-1145, 2008.

[42] L. Su, W. Wang, and H. Wang, "A characteristic difference method for the transient fractional convection-diffusion equations," Applied Numerical Mathematics, vol. 61, no. 8, pp. 946960, 2011.

[43] S. B. Yuste, "Weighted average finite difference methods for fractional diffusion equations," Journal of Computational Physics, vol. 216, no. 1, pp. 264-274, 2006. 


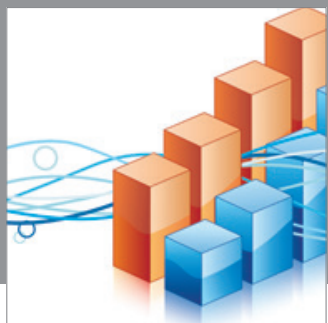

Advances in

Operations Research

mansans

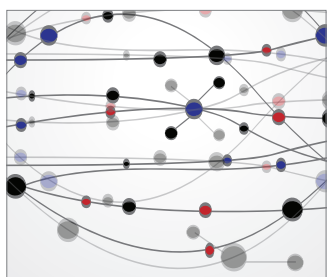

The Scientific World Journal
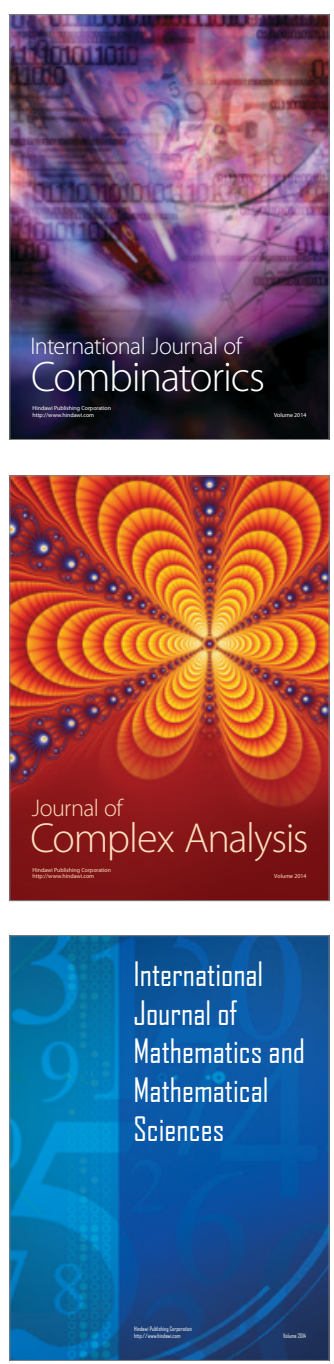
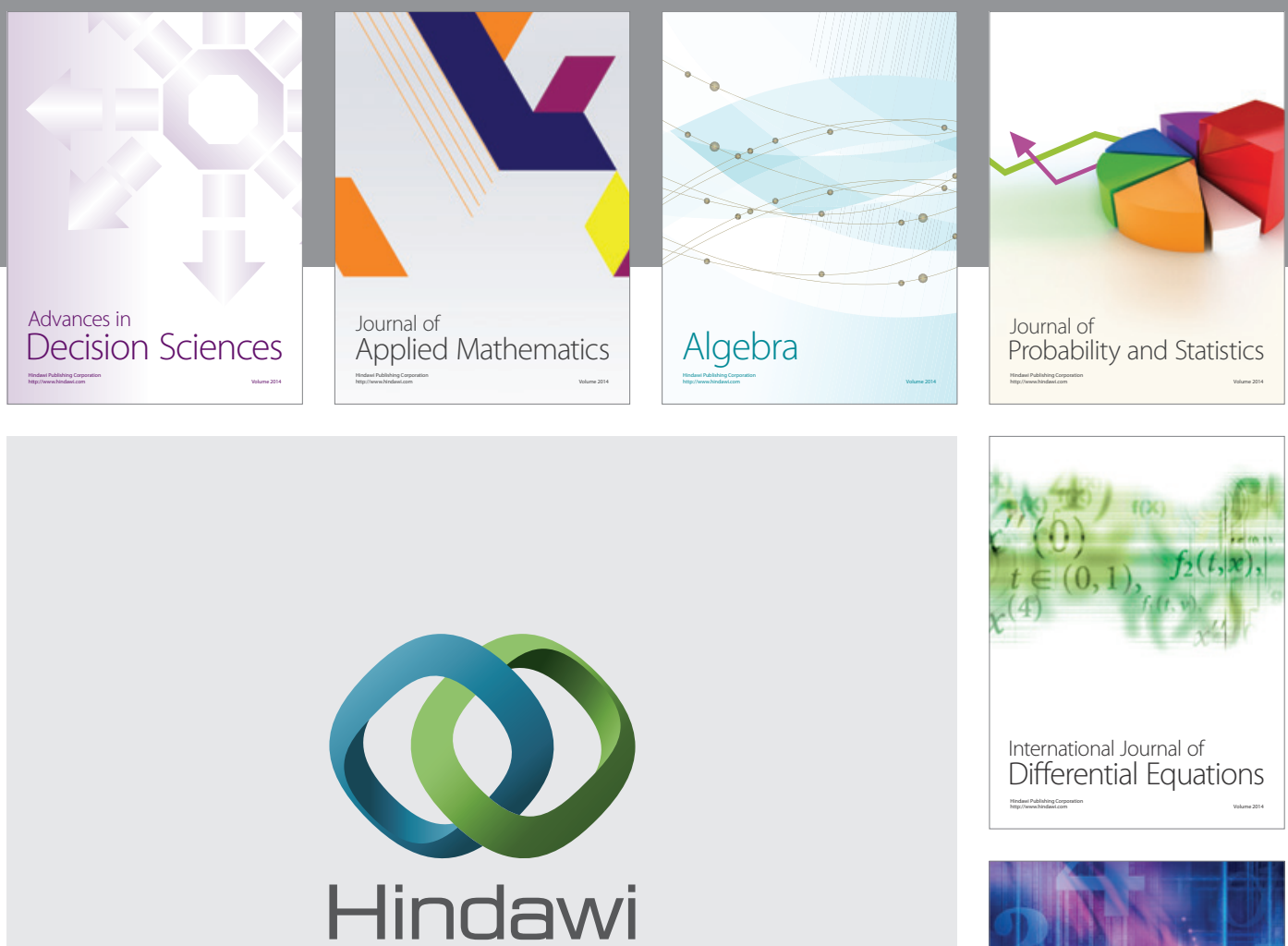

Submit your manuscripts at http://www.hindawi.com
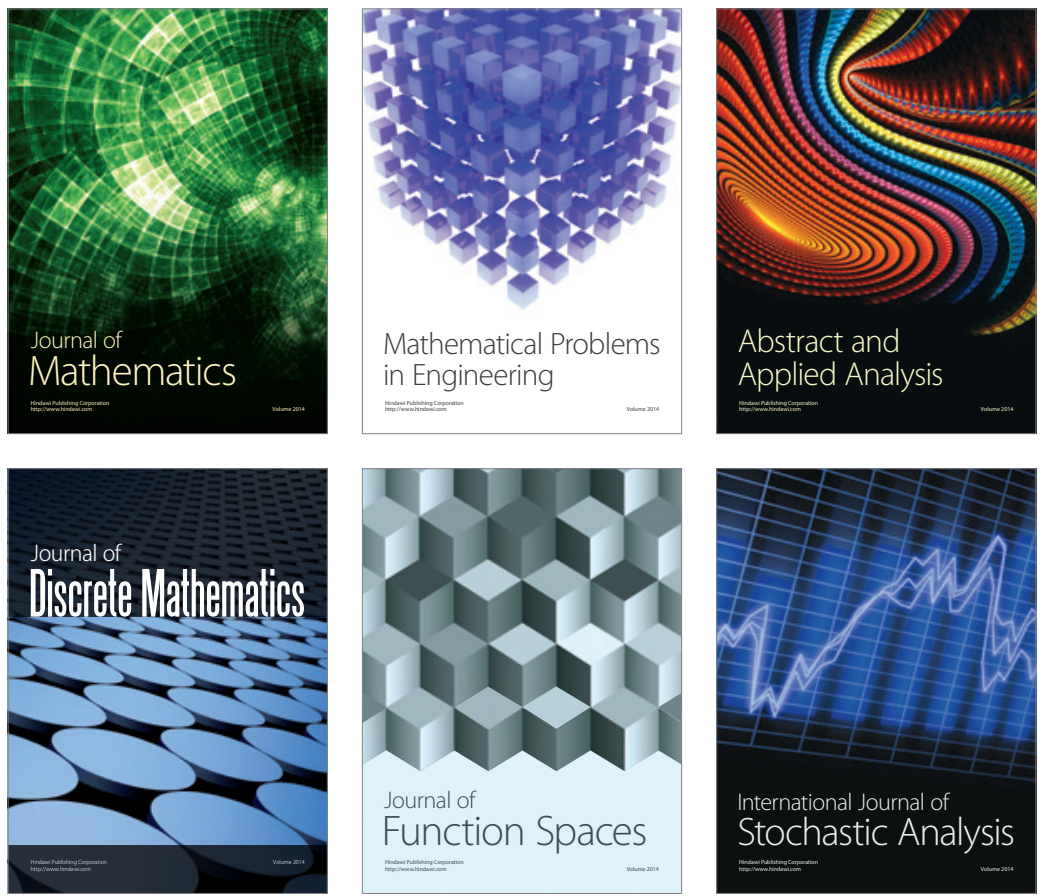

Journal of

Function Spaces

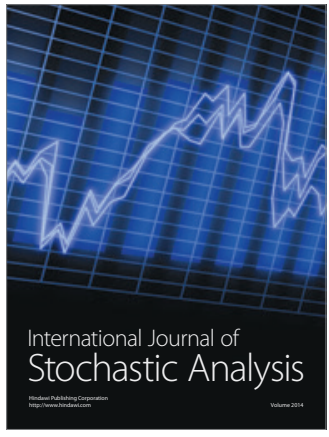

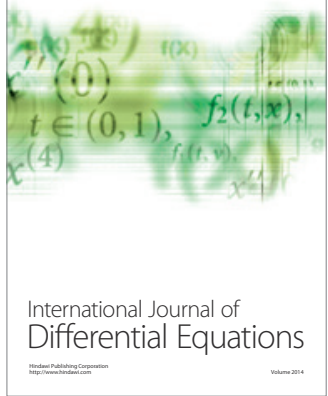
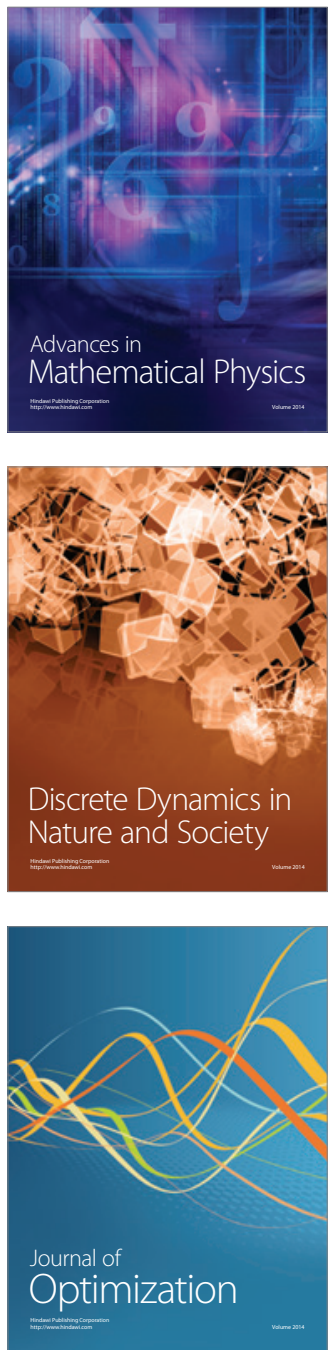